American Medical Journal 3 (2): 231-236, 2012

ISSN 1949-0070

(C) 2012 Science Publications

\title{
Assessment of Risk of Falls in Elderly Patients with Osteoarthritis of the Knee and Low Back Pain
}

\author{
${ }^{1}$ Polianna Delfino Pereira, ${ }^{2}$ Rodrigo Miranda, ${ }^{3,4}$ Marco Orsini, \\ ${ }^{3}$ Marco Antonio Araujo Leite, ${ }^{5}$ Victor Hugo do Vale Bastos and ${ }^{5}$ Dionis Machado \\ ${ }^{1}$ Department of Neuroscience and Behavioral Sciences, \\ School of Medicine of Ribeirao Preto (USP), Ribeirao Preto, SP, Brazil \\ ${ }^{2}$ Department of Physiotherapist, \\ Federal University of the Valleys of the Jequitinhonha and Mucuri (UFVJM), \\ Diamantina, MG, Brazil \\ ${ }^{3}$ Department of Neurology, School of Medicine, \\ Fluminense Federal University (UFF), Niteroi, RJ, Brazil \\ ${ }^{4}$ Masters of Program the Science Rehabilitation, \\ University Canter Augusto Motta (UNISUAM), Rio de Janeiro, RJ, Brazil \\ ${ }^{5}$ Department of Physiotherapist, Federal University of Piaui (UFPI), Parnaiba, PI, Brazil
}

\begin{abstract}
This study aimed identify which groups of elderly are at higher risk of falls, with osteoarthritis (OA), low back pain or both conditions. 29 elderly were distributed in three groups: OA of the Knee (OAK), Low Back Pain (LBP) and the two events concomitantly (OL). Evaluation tools applied were: Mini Mental State Examination (MMSE), Visual Analogue Scale (VAS), Western Ontario and McMaster Universities Osteoarthritis Index (WOMAC), Roland Morris Functional Performance Questionnaire, Gait Speed (GS), Timed Get Up and Go Test (TGUGT) and Berg Balance Scale (BBS). No differences were found in the three groups for assessment by GS, TGUGT and BBS instruments. A significative difference was found by RMQ in groups LPB and OL, WOMAC found difference in pain domain between groups OL and OAK and VAS analysis showed significative difference between group OL to the others groups. No significative difference was found between groups by the assessment of balance.
\end{abstract}

Key words: Berg Balance Scale (BBS), Gait Speed (GS), Visual Analogue Scale (VAS), Mini Mental State Examination (MMSE), Low Back Pain (LBP)

\section{INTRODUCTION}

The increasing life expectancy in the wordwide has demanded greater efficiency in elderly care (ValimRogatto et al., 2009; Avelar et al., 2010; 2011; Tsonga et al., 2011). The demographic transition also causes significant changes in epidemiologic profile, changing the model of health characterized by infections contagious diseases to a higher proportion of chronic degenerative diseases (Valim-Rogatto et al., 2009; Gananca et al., 2010; Maraschin et al., 2010; Avelar et al., 2011; Falsarella et al., 2011; Kao et al., 2011). A requirement for maintaining a healthy aging is the maintenance of functional capacity, wich englobes individual independence and his active participation in society. Falls are factors that may contribute to reduce functional capacity and can cause significant consequences as disability, health impairment, changes in quality of life, imobility, restrictions of activities, institutionalization, psychological damage and death (Pedrinelli et al., 2009; Valim-Rogatto et al., 2009; Maraschin et al., 2010; Charansonney, 2011). A fall may be defined as an unintentional event that results in a change of individual's position to a lower level in relation to its initial position, without skilled time correction (Gine-Garriga et al., 2009; Queiroz et al., 2009; Alvares et al., 2010; Chen et al., 2010; Ferreira and Yoshitome, 2010; Ricci et al., 2010; Charansonney, 2011; Correa et al., 2012). This event can be caused by several intrinsic and extrinsic factors, creating a multicausal phenomenon in wich can be detached the presence of comorbidities as intrinsic factors (ValimRogatto et al., 2009; Ferreira and Yoshitome, 2010; Paula et al., 2010; Ricci et al., 2010). 
With advancing age, a chronic degenerative disease, the Osteoarthritis (OA), usually is detected. The knees are the joints most often affected and for their relevance in corporal biomechanics it may trigger a negative impact on functionality. Like presence of knee pain, characteristic of $\mathrm{OA}$, back pain is also usually common and these symptoms can reflect on quality of life of elderly people (Pedrinelli et al., 2009; Valim-Rogatto et al., 2009; Avelar et al., 2010; 2011). The manifestation of low back pain is characterized by painful sensations and discomfort among the inferior gluteal line and costal border that can irradiated to the lower limbs (LL). Low back pain is also associated with restrictions of many activities, decrease in functional capacity and can lead to falls (Maraschin et al., 2010; Ricci et al., 2010).

Falls usually are associated with aging and this situation constitute a great problem in public health due high costs, physical and psychological consequences (Valim-Rogatto et al., 2009). In this way, it is necessary to identify the relationship between falls and conditions like OA and low back pain to guide elderly people which are affected by them. In addition, the risk of falls may be minimized with such information. This study aimed identify which groups of elderly patients are at higher risk of falls, the patients with OA, low back pain or both conditions.

\section{MATERIALS AND METHODS}

This study is according with ethical principles for research involving human as described by the Declaration of Helsinki and was approved by the Ethics Committee of Federal University of the Jequitinhonha and Mucuri Valleys. It was a clinical cross-sectional study and the sample was obtained by verbal invitation on the Association of Retirees and Pensioners of Diamantine/MG. The study included elderly aged over 60 years, both sexes, living in Diamantine/MG, with diagnosis of $\mathrm{OA}$ in at least one knee, based on clinical criteria of American College of Rheumatology, clinical manifestation of low back pain or both conditions, which were not in physical therapy, who had not recent trauma at knees or spine and that present medical conditions and cognitive requirements for the aplication of balance assessment tools (Avelar et al., 2010; 2011; Falsarella et al., 2011; Kao et al., 2011).

Exclusion criteria were patients with uncompensated cardiovascular, neurological or psychiatric disease, amputation of lower limbs, unable to stand up alone without another person or device aid, severe visual or hearing impairment uncorrected, using medications that may interfere in balance and subjects who had no sphincter control (urinary or fecal). Were contacted 90 volunteers, of whom 61 did not participate due exclusion criteria or lack of interest? In this way 29 volunteers constituted the sample and they were divided into three groups: ten subjects in knee OA group $(\mathrm{OAK})$, nine in Low Back Pain group (LBP) and ten subjects in knee OA and low back pain group (OL).

Subjects received instructions to proceed on the assessment day, such as use bathroom before tests, wear comfortable clothes and usual shoes, drink water before, make a light meal or snack, avoid strenuous activities for at least $2 \mathrm{~h}$ before exam and no caffeine $24 \mathrm{~h}$. before the assessment. These instructions were given in order to avoid any influence on the outcome of this study (Tarnopolsky, 2010; Avelar et al., 2011; Santos et al., 2011b).

All subjects were informed about the nature of this research and were invited to sign the Term of Informed Consent. For the assessment the volunteer was conducted to a private room where tests were done for identification of low back pain and to confirme the clinical criteria for OA based on American College of Rheumatology. A physical therapy evaluation form was applied to register data of each volunteer (Avelar et al., 2010; 2011; Falsarella et al., 2011; Kao et al., 2011). The evaluation tools applied were: Mini Mental State Examination (MMSE), Visual Analogue Scale (VAS), Western Ontario and McMaster Universities Osteoarthritis Index (WOMAC) for subjects with OA (OAK and OL groups), Roland Morris Functional Performance Questionnaire (only for LBP and OL groups), Gait Speed (GS), Timed Get Up and Go Test (TGUGT) and Berg Balance Scale (BBS).

The MMSE is an important tool for cognitive screening and in this study was used the scores considering educational level of subjects (Avelar et al., 2011; Karuka et al., 2011). The Roland Morris Questionnaire (RMQ) consists of 24 items with scores zero or one (no or yes, respectively), to each one and demonstrates functional consequences resulting from low back pain. For example, score above 14 indicates subject inability. The WOMAC applied in OAK and OL groups in order to verify level of pain, stiffness and physical function (functionality) perceived by volunteers in $72 \mathrm{~h}$ before the assessment. As higher score worse are the symptoms (Avelar et al., 2010; 2011; Falsarella et al., 2011; Neto et al., 2011; Kao et $a l ., 2011)$. The VAS was used to assess the perception of pain in $24 \mathrm{~h}$ prior the exam. The subject was required to mark his level of pain in a line of ten $\mathrm{cm}$. The highest level of pain is near from the end of the line $(10 \mathrm{~cm})$. 
GS was calculated in a route of $10 \mathrm{~m}$, but the $2 \mathrm{~m}$ initial and final were disconsidered due its relationship with acceleration and deceleration. In this way, GS was calculated dividing the time spent for this activity by 6 $\mathrm{m}$. In this study, GS lower $1 \mathrm{~m} \mathrm{~s}^{-1}$ was considered as risk of falls (Salminen et al., 2009; Novaes et al., 2011; Tsonga et al., 2011). TGUGT assesses sitting balance, transfer (sitting/ standing and standing/sitting), stability during walking and changing direction. The time spent on this activity is associated with subject's performance. The scores indicate functional mobility, for example: Executing TGUGT in ten seconds or less indicates functional independence and no risk of falls, among 11 and 20 seconds indicates parcial dependence as a moderate risk of falls and above 20 sec indicates functional dependence in many activities of daily living and a high risk of falls (Queiroz et al., 2009; Gine-Garriga et al., 2009; Chen et al., 2010; Karuka et al., 2011; Avelar et al., 2011; Silva et al., 2011). The BBS evaluates postural control necessary to some activities. Each task can be punctuated from zero (unable to perform the task) to four (performing task independently). Higher scores indicate better performance (Avelar et al., 2010; Karuka et al., 2011).

A descriptive statistics was implemented in order to provide measures of central tendency and variability of the sample. The Shapiro-Wilk test was used to detected Gaussian distribuition for the variables: age, MMSE, RMQ, WOMAC and GS. An ANOVA (one way), with Tukey Post-Hoc test for data with normal distribuition, was employed to compare means by the three groups (OAK, LBP and OL). The non parametric Kruskal Wallis test was applied to the variables that have not normal distribuition (BBS and TGUGT). Data were analyzed using SPSS 19.0. For all cases was considered $\mathrm{p} \leq 0.05$.

\section{RESULTS}

The sample of this study was 29 volunteers aged between 60 and 86 years. The average age was $70.10 \pm 6.6$ years. In each group the average age was: $70.88 \pm 8.0$ in LBP group, 71.30 \pm 5.5 in OAK group and $68.20 \pm 6.4$ in OL group. The MMSE mean was $25.27 \pm 2.68$ points and the average of punctuation in groups LBP, OAK and OL was respectively: $25.22 \pm 1.48, \quad 26.00 \pm 3.05$ and $24.60 \pm 3.16$. There was no significant difference in sample for age $(\mathrm{p}=0.54$ and $\mathrm{F}[26.2]=0.62)$ and for MMSE punctuation $(\mathrm{p}=0.52$ and $\mathrm{F}[26.2]=0.66)$.
Table 1: Values obtained by each group in the application of the assessment tools

\begin{tabular}{llcll}
\hline Variable & LBP & OAK & OL & $\mathrm{p}$ \\
\hline Roland Morris & $7.88 \pm 4.53$ & - & $15.10 \pm 4.53$ & $0.003^{*}$ \\
WOMAC & & & & \\
- Pain & - & $28.00 \pm 17.82$ & $41.50 \pm 13.55$ & $0.038^{*}$ \\
- Stiffness & - & $32.00 \pm 27.17$ & $39.50 \pm 18.92$ & 0.483 \\
- Physical Function & - & $30.28 \pm 22.05$ & $43.67 \pm 15.68$ & 0.135 \\
VAS & $3.55 \pm 2.600$ & $3.10 \pm 2.9900$ & $6.30 \pm 2.7900$ & $0.037^{*}$ \\
GS & $1.32 \pm 0.320$ & $1.34 \pm 0.3500$ & $1.31 \pm 0.2100$ & 0.967 \\
TGUGT & $9.27 \pm 2.100$ & $9.54 \pm 2.3300$ & $10.02 \pm 2.240$ & 0.705 \\
BBS & $52.44 \pm 4.66$ & $50.80 \pm 4.360$ & $49.70 \pm 3.650$ & 0.109 \\
\hline
\end{tabular}

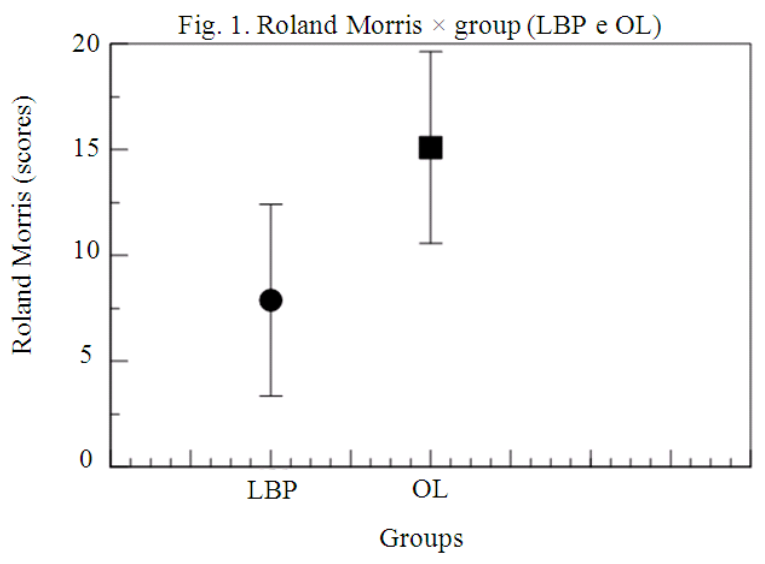

Fig. 1: Significative difference between groups LPB and OL on the scores of RMQ

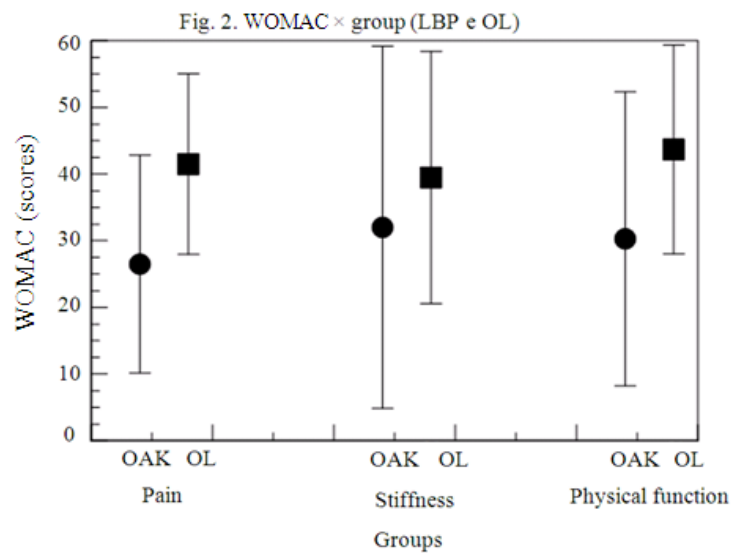

Fig. 2: Significative difference between groups OAK and OL only on WOMAC pain domain

In this study, sample was mostly composed by female individuals (84\%). $41,37 \%$ of total sample presents with comorbities and in groups LBP, OAK and OL respectively 70, 66.67 and $90 \%$ of volunteers performed physical activity for at least thirty minutes three times a week. 


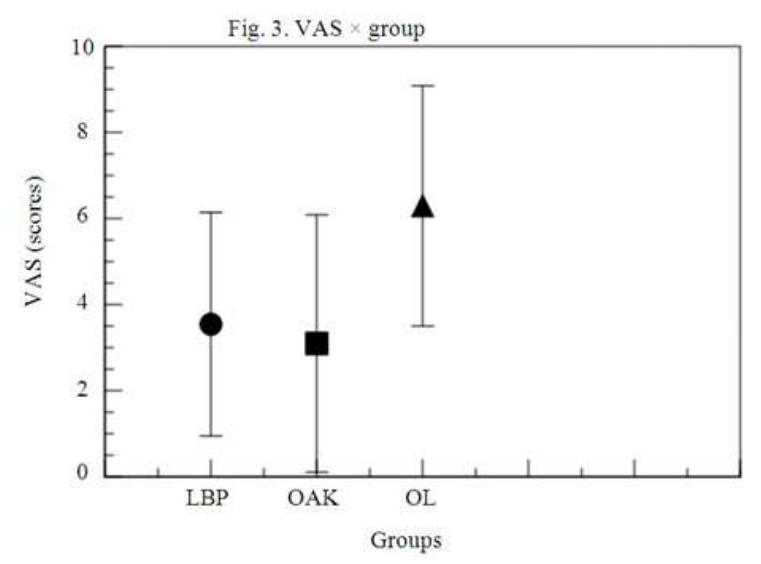

Fig. 3: OL Group showed a significative difference level of pain on VAS compared to the others groups

The values obtained by each group in the application of the assessment tools were listed in Table 1.

Statistical analysis identified no significant differences in the three groups for the assessment by GS, TGUGT and BBS instruments. A significative difference was found by RMQ in groups LPB and OL (Fig. 1), the WOMAC found difference only in pain domain (Fig. 2) between groups OL and OAK and the analysis of VAS shows significative difference between groups OL to the others groups (Fig. 3).

\section{DISCUSSION}

This study aimed to determine which groups of elderly patients with OA, low back pain and two concurrent events are at higher risk of falls. For this was used a selection of instruments and questionnaires easily reproducible, with copyright or public domain and that present low cost and good reliability. In this sample was considered elderly above 60 years because the study was conducted in Brazil that is considered a country in development. The sample composition was consisted predominantly of female volunteers as can be seen in other studies and indicates a feminization process of the population that become more evident with advancing age due female longer life expectancy (Alvares et al., 2010; Ferreira and Yoshitome, 2010; Maraschin et al., 2010; Paula et al., 2010; Silva et al., 2011; Tsonga et al., 2011).

Low back pain can cause changes in subject's functional capacity, in physical performance and may be a factor in risk to falls. A research demonstrated low correlation between low back pain and functional performance measure (Ocarino et al., 2009), but in this present study was found a significative difference by RMQ in OL group that sugests low back pain has a negative impact into functional performance. In this context, the RMQ application showed worse functional performance in elderly people who presents the two events (OA and low back pain) and this may predispose them to risk of falls (Ricci et al., 2010).

The clinicals criteria for OA of American College of Rheumatology consist of pain, stiffness, crackle, age over 38 years and bony enlargement to the physic exam.

The literature indicates that $\mathrm{OA}$ causes higher functional limitation in women and represent a risk of falls 2.4 times higher than in a elderly without OA. The data of the present study showed significative difference only in pain domain of WOMAC for the OL group. This is consistent with studies that show pain as main symptom of $\mathrm{OA}$ and its association with functional inability and falls (Avelar et al., 2010; Santos et al., 2011a; Falsarella et al., 2011; Kao et al., 2011). Furthermore, OL group presents a significative difference in level of pain by VAS, subjects in OL group showed a higer level of pain in contrast with the others groups.

Presence of pain may trigger antalgic postures and lameness that affect posture, gait and balance, especially in elderly. According a study by Ricci et al. (2010) and Cruz et al. (2011) subjects with pain have a higher risk of falling. Another study aimed verifies if chronic pain could lead to a reduction of functional capacity, predisposing elderly community to a higher risk of falling. The authors concluded that chronic pain is associated to a higher risk (Leveille et al., 2009). The results of the present study indicate that OL group presented higher scores in contrast with the others groups, suggesting a higher risk of falls to this group.

Balance in elderly is often stricken and to maintain stability usually they reduce GS as well as step length (Gine-Garriga et al., 2009; Queiroz et al., 2009; Chen et al., 2010; Novaes et al., 2011). A confortable speed to gait in elderly is decribed as $0.60-1.45 \mathrm{~m} \mathrm{~s}^{-1}$. The volunteers in this study showed $1.32 \mathrm{~m} \mathrm{~s}^{-1}$ of average in GS, it means a pattern without risk of falls (Salminen et al., 2009; Novaes et al., 2011; Tsonga et al., 2011). The present study do not observed differences in TGUGT between groups and TGUGT average was $9.62 \mathrm{sec}$, time considered normal for healthful adults. As described in literature, , this instrument is an useful tool to evaluate changes of balance in healthy elderly (GineGarriga et al., 2009; Goncalves et al., 2009; Queiroz et al., 2009; Chen et al., 2010; Avelar et al., 2011; 
Karuka et al., 2011). Some studies indicate correlation between BBS and other functionals measures such as the TGUGT and GS. Sample in this study had mean score in BBS of 50.93 points and there was no difference between groups.

Possible reasons for the results of the present study may be some characteristics of the sample, as follows: actives subjects, without visual changes, few comorbities and no cognitive impairment. Many researches indicate that physical activity produces positive effects on overall health of subjects, ensuring normals levels of physical, mental and social health. Moreover, physical activity provides higher functional independence, autonomy and quality of life (Paula et al., 2010; Ricci et al., 2010; Charansonney, 2011; Ozturk et al., 2011; Silva et al., 2011). Another special characteristic in this sample was few comorbities. Some studies indicate that number of comorbities is directly proportional to the risk of falls i.e., as higher the number of comorbities higher will be the risk of falls (Gine-Garriga et al., 2009; Queiroz et al., 2009; Alvares et al., 2010; Chen et al., 2010; Cruz et al., 2011; Ferreira and Yoshitome, 2010; Ricci et al., 2010; Silva et al., 2011). Cognitive impairment also can increase risk of falls because it usually decreased anticipatory responses, causes spatial disorientation, changes in gait, posture and balance (Alvares et al., 2010; Ricci et al., 2010; Silva et al., 2011). In this study, a exclusion criteria was cognitive decline that was measured by MMSE. Thus, in this sample there was no cognitive impairment that could damage the subject performance.

\section{CONCLUSION}

It may be conclude that in this study there was found no significative difference between groups with the assessment of balance by instruments used, but group OL (that presented the two events: OA in knees and low back pain) showed worst performance than others groups and this may be represent a higher predisposition to falls. Furthermore the sample was fairly homogeneous and presents few factors of risk to falls. Some recomendations to next studies consisting in larger sample, a longitudinal study and a control group.

\section{REFERENCES}

Alvares, L.M., R.D.C. Lima and R.A.D. Silva, 2010. Falls by elderly people living in long-term care institutions in Pelotas, Rio Grande do Sul State, Brazil. Cad. Saude Publica, 26: 31-40.
Avelar, N.C., A.P. Simao, R. Tossige-Gomes, C.D.C. Neves and E. Rocha-Vieira et al., 2011. The effect of adding whole-body vibration to squat training on the functional performance and self-report of disease status in elderly patients with knee osteoarthritis: A randomized, controlled clinical study. J. Altern. Complement Med., 17: 11491155. PMID: 22087576

Avelar, N.C.P., A.C. Bastone, M.A. Alcântara and W.F. Gomes, 2010. Effectiveness of aquatic and nonaquatic lower limb muscles endurance training in the static and dynamic balance of elderly people. Rev. Bras. Fisioter, 14: 229-236. DOI: 10.1590/S1413-35552010000300007

Charansonney, O.L., 2011. Physical activity and aging: a life-long story. Discov Med., 12: 177-185. PMID: 21955845

Chen, Y.M., Y.W. Chuang, S.C. Liao, Y.J. Tang and J.J. Tsai et al., 2010. Predictors of functional recovery (FR) for elderly hospitalized patients in a Geriatric Evaluation and Management Unit (GEMU) in Taiwan. Arch Gerontol. Geriatr., 50: S1-S5. PMID: 20171448

Correa, A.D., I.A. Marques, M.C. Martinez, P.S. Laurino and E.R. Leao et al., 2012. The implementation of a hospital's fall management protocol: Results of a four-year follow-up. Rev. Esc. Enferm., 46: 67-74. PMID: 22441267

Cruz, H.M.F., C.A.M. Pimenta, M.S.G. Dellarozza, P.E. Braga and M.L. Lebrao et al., 2011. Falls in chronic pain elderly patients: prevalence and associated factors. Rev. Dor., 12: 108-14. DOI: 10.1590/S1806-00132011000200006

Falsarella, G.R., I.B. Coimbra, A.L. Neri, C.C. Bracelos and L.T. Costallat et al., 2011. Impact of rheumatic diseases and chronic joint symptoms on quality of life in the elderly. Arch. Gerontol. Geriatr., 54: e77-e82. PMID: 21871677

Ferreira, D.C. and A.Y. Yoshitome, 2010. Prevalence and features of falls of institutionalized elders. Rev. Bras. Enferm., 63: 991-997. PMID: 21308234

Gananca, F.F., J.M. Gazzola, C.F. Gananca, H.H. Caovilla and M.M. Gananca et al., 2010. Elderly falls associated with benign paroxysmal positional vertigo. Braz. J. Otorhinolaryngol., 76: 113-120. PMID: 20339699

Gine-Garriga, M., M. Guerra, M.M.D. Olmo, C. Martin and V.B. Unnithan, 2009. Sensitivity of a modified version of the 'timed get up and go' test to predict fall risk in the elderly: A pilot study. Arch. Gerontol. Geriatr., 49: e60-66. PMID: 18977044 
Goncalves, D.F.F., N.A. Ricci and A.M.V. Coimbra, 2009. Functional balance among communitydwelling older adults: A comparison of their history of falls. Rev. Bras. Fisioter., 13: 316-323.

Kao, M.J., M.P. Wu, M.W. Tsai, W.W. Chang and S.F. $\mathrm{Wu}, 2011$. The effectiveness of a self-management program on quality of life for knee osteoarthritis (OA) patients. Arch. Gerontol. Geriatr., 54: 317324. DOI: 10.1016/j.archger.2011.05.018

Karuka, A.H., J.A. Silva and M.T. Navega, 2011. Analysis of agreement of assessment tools of body balance in the elderly. Rev. Bras. Fisioter., 15: 460466. PMID: 22218711

Leveille, S.G., R.N. Jones, D.K. Kiely, J.M. Hausdorff and R.H. Shmerling et al., 2009. Chronic musculoskeletal pain and the occurrence of falls in an older population. JAMA, 302: 2214-2221. PMID: 19934422

Maraschin, R., P.S. Vieira, C.P. Leguisamo, F. Dal'vesco and J.P. Santi, 2010. Low back pain and pain in the lower extremity in aged: Etiology in review. Fisioter. Mov. (Impr.)., 23: 627-639. DOI: 10.1590/S0103-51502010000400013

Neto, E.M.D.F., T.T. Queluz and B.F.A Freire, 2011. Physical activity and its association with quality of life in patients with osteoarthritis. Rev. Bras. Reumatol., 51: 539-549.

Novaes, R.D., A.S. Miranda. and V.Z. Dourado, 2011. Velocidade usual da marcha em brasileiros de meia idade e idosos. Rev. Bras. Fisioter., 15: 117-122. DOI: 10.1590/S141335552011000200006

Ocarino, J.M., G.G.P. Goncalves, D.V. Vaz, A.A.V. Cabral and J.V. Porto et al., 2009. Correlation between a functional performance questionnaire and physical capability tests among patients with low back pain. Rev. Bras. Fisioter., 13: 343-349. DOI: $10.1590 /$ S1413-35552009005000046

Ozturk, A., T.T. Simsek, E.T. Yumin, M. Sertel and M. Yumim, 2011. The relationship between physical, functional capacity and Quality of Life (QoL) among elderly people with a chronic disease. Arch. Gerontol. Geriatr., 53: 278-283. PMID: 21215469

Paula, F.L., M.J.M. Fonseca, R.V.C. Oliveira and S. Rozenfeld, 2010. Profile of elderly admitted to public hospitals of Niteroi (RJ) due to falls. Rev. Bras. Epidemiol., 13: 587-595. DOI: 10.1590/S1415-790X2010000400004
Pedrinelli, A., L.E. Garcez-Leme and R.S.A. Nobre, 2009. The effect of physical training on locomotive apparatus in elderly people. Rev. Bras. Ortop., 44: 96-101. DOI: 10.1590/S0102-36162009000200002

Queiroz, L., S. Lira and A. Sasaki, 2009. Identificating the risk of falls through functional mobility assessmentin hospitalized elderly. Rev. Baiana Saúde Pub., 33: 534-543.

Ricci, N.A., D.F.F. Goncalves, I.B. Coimbra and A.M.V. Coimbra, 2010. Factors associated with the history of falls of elderly assisted by the family health program. Saúde Soc., 19: 898-909.

Salminen, M., T. Vahlberg, S. Sihvonen, N. Sjösten and M. Piirtola et al., 2009. Effects of risk-based multifactorial fall prevention on postural balance in the community-dwelling aged: A randomized controlled trial. Arch. Gerontol. Geriatr., 48: 22-27. DOI: 10.1016/j.archger.2007.09.006

Santos, F.C., P.M.R.D. Souza, J.T. Neto and A.N. Atallah, 2011a. Treatment of pain associated to knee osteoarthritis in the elderly: A randomized double-blind clinical trial with lysine clonixinate. Rev. Dor., 12: 6-14. DOI: 10.1590/S180600132011000100003

Santos, M.L., W.F. Gomes, D.S. Pereira, D.M. Oliveira and J.M. Dias et al., 2011b. Muscle strength, muscle balance, physical function and plasma interleukin-6 (IL-6) levels in elderly women with knee osteoarthritis (OA). Arch. Gerontol. Geriatr., 52: 322-326. PMID: 20627334

Silva, E.C., N.B. Duarte and P.M.M. Arantes, 2011. Study of relationship between level of physical activity and risk of falls in older women. Fisioter. Pesq., 18: 23-30.

Tarnopolsky, M.A., 2010. Caffeine and creatine use in sport. Ann. Nutr. Metab., 57: 1-8. DOI: 10.1159/000322696

Tsonga, T., S. Kapetanakis, C. Papadopoulos, J. Papathanasiou and N. Mourgias et al., 2011. Evaluation of improvement in quality of life and physical activity after total knee arthroplasty in greek elderly women. Open Orthop. J., 5: 343-347. DOI: $10.2174 / 1874325001105010343$

Valim-Rogatto, P.C., G.P. Rogatto, A.C.P. Corrêa and A.C.P. Brêtas, 2009. Level of physical activity and accidental falls in elderly: A systematic review. Rev. Bras. Cineantropom. Desempenho Hum., 11: 235-242. 